OPEN ACCESS

Edited by: John W. Austin,

Health Canada, Canada

Reviewed by: Yi Wang,

Auburn University, United States Bo Segerman, National Veterinary Institute, Sweden Francois Wasels,

IFP Energies nouvelles, France

${ }^{*}$ Correspondence: Miia Lindström miia.lindstrom@helsinki.fi

tThese authors have contributed equally to this work

Specialty section: This article was submitted to Food Microbiology, a section of the journal Frontiers in Microbiology

Received: 14 October 2020 Accepted: 05 January 2021 Published: 27 January 2021

Citation:

Mertaoja A, Nowakowska MB, Mascher G, Heljanko V, Groothuis D, Minton NP and Lindström M (2021)

CRISPR-Cas9-Based Toolkit for Clostridium botulinum Group /I Spore and Sporulation Research.

Front. Microbiol. 12:617269. doi: 10.3389/fmicb.2021.617269

\section{CRISPR-Cas9-Based Toolkit for Clostridium botulinum Group II Spore and Sporulation Research}

\author{
Anna Mertaoja ${ }^{1 \dagger}$, Maria B. Nowakowska ${ }^{1 \dagger}$, Gerald Mascher ${ }^{1}$, Viivi Heljanko', \\ Daphne Groothuis ${ }^{2}$, Nigel P. Minton ${ }^{2}$ and Miia Lindström ${ }^{1 \star}$
}

\begin{abstract}
${ }^{1}$ Department of Food Hygiene and Environmental Health, Faculty of Veterinary Medicine, University of Helsinki, Helsinki, Finland, ${ }^{2}$ Clostridia Research Group, BBSRC/EPSRC Synthetic Biology Research Centre (SBRC), Biodiscovery Institute, School of Life Sciences, University of Nottingham, Nottingham, United Kingdom
\end{abstract}

The spores of Clostridium botulinum Group II strains pose a significant threat to the safety of modern packaged foods due to the risk of their survival in pasteurization and their ability to germinate into neurotoxigenic cultures at refrigeration temperatures. Moreover, spores are the infectious agents in wound botulism, infant botulism, and intestinal toxemia in adults. The identification of factors that contribute to spore formation is, therefore, essential to the development of strategies to control related health risks. Accordingly, development of a straightforward and versatile gene manipulation tool and an efficient sporulation-promoting medium is pivotal. Our strategy was to employ CRISPR-Cas9 and homology-directed repair (HDR) to replace targeted genes with mutant alleles incorporating a unique 24-nt "bookmark" sequence that could act as a single guide RNA (sgRNA) target for Cas9. Following the generation of the sporulation mutant, the presence of the bookmark allowed rapid generation of a complemented strain, in which the mutant allele was replaced with a functional copy of the deleted gene using CRISPR-Cas9 and the requisite sgRNA. Then, we selected the most appropriate medium for sporulation studies in $C$. botulinum Group II strains by measuring the efficiency of spore formation in seven different media. The most effective medium was exploited to confirm the involvement of a candidate gene in the sporulation process. Using the devised sporulation medium, subsequent comparisons of the sporulation efficiency of the wild type (WT), mutant and "bookmark"-complemented strain allowed the assignment of any defective sporulation phenotype to the mutation made. As a strain generated by complementation with the WT gene in the original locus would be indistinguishable from the parental strain, the gene utilized in complementation studies was altered to contain a unique "watermark" through the introduction of silent nucleotide changes. The mutagenesis system and the devised sporulation medium provide a solid basis for gaining a deeper understanding of spore formation in C. botulinum, a prerequisite for the development of novel strategies for spore control and related food safety and public health risk management.

Keywords: Clostridium botulinum Group II, CRISPR-Cas9, sporulation medium, spoOA, spore 


\section{INTRODUCTION}

Clostridium botulinum is a Gram-positive, strictly anaerobic, spore-forming bacterium. C. botulinum strains are traditionally classified into four genetically and physiologically diverse groups designated I-IV (Hatheway, 1993). The critical characteristic of all four groups is their ability to produce extremely potent neurotoxin which is the causative agent of botulism, a rare but deadly disease affecting humans and animals (Sobel, 2005). C. botulinum strains of Group I and Group II are usually associated with human botulism, which classically manifests as a food poisoning. This form of botulism results from consumption of foods where C. botulinum spores germinated and outgrew into a neurotoxigenic culture. Their toxinogenic potential at refrigeration temperatures makes C. botulinum Group II strains a particular food safety concern (Lindström et al., 2006). Moreover, C. botulinum spores can colonize wounds or the digestive tract of infants and susceptible adults with compromised gut microbiota, causing the toxicoinfectious forms of wound and intestinal botulism, respectively (Harris, 2015).

Any interventions for controlling the risks posed by C. botulinum spores rely on the fundamental understanding of the spore composition and resistance properties, as well as the processes of spore formation, spore germination, and neurotoxin production. High-quality mechanistic research is reliant on precise deletions, genome manipulations, and efficient screening assays for the targeted phenotypes. Some progress has been made in recent times as a consequence of the development of appropriate genome editing tools (Heap et al., 2007; Ng et al., 2013; Minton et al., 2016; Cañadas et al., 2019; Ingle et al., 2019; Kuehne et al., 2019). These include the extensively utilized ClosTron together with various allelic exchange methodologies which have been exploited to successfully construct insertion and deletion mutants of sporulation and toxin-related genes in C. botulinum (Dahlsten et al., 2013; Kirk et al., 2014; Mascher et al., 2014, 2017; Zhang et al., 2014; Clauwers et al., 2016, 2017). The ClosTron, however, like any insertional mutagen is compromised by the fact that mutants may have altered phenotypes due to polar effects on genes located downstream of the intron insertion (Heap et al., 2007). Although polar effects may be overcome using allelic exchange to make in-frame, markerless deletion mutants (reviewed in Minton et al., 2016), the strategy utilized so far in C. botulinum Group II strains has resulted in the insertion of an antibiotic resistance gene (Clauwers et al., 2016, 2017). To make markerless deletions, counterselection markers are necessary, such as pyrE and $\operatorname{codA}$ (Minton et al., 2016), but their deployment requires the use of minimal media. Finding a minimal medium that is applicable to metabolically diverse C. botulinum strains is, however, challenging (Whitmer and Johnson, 1988).

Recently, the ease with which allelic exchange may be deployed in clostridia, including C. botulinum Group I strains, has been considerably improved through the exploitation of clustered regularly interspaced short palindromic repeats-CRISPRassociated protein 9 (CRISPR-Cas9; Cañadas et al., 2019; Ingle et al., 2019; McAllister and Sorg, 2019). Using such an approach, the allelic-exchange driven gene deletion makes the resulting mutant cells immune to Cas9-cleavage. This enables direct selection of mutant colonies, as opposed to having to be passaged onto specialized media where counterselection markers are employed. Consequently, the reduced number of steps leading to the mutant isolation decreases the likelihood of accumulating undesired ancillary mutations [single nucleotide polymorphisms (SNPs) and/or insertion/deletions (InDels)] that may affect phenotype. Thus, CRISPR-Cas9-based methods offer distinct advantages for generating mutants in C. botulinum Group II strains.

Another critical component in the fundamental spore research is a medium that efficiently promotes sporulation and ensures sufficient spore yields for downstream applications. While Bacillus spp. sporulation is mainly induced by starvation (Grossman and Losick, 1988), the factors triggering clostridial sporulation appear more complex (Perkins and Tsuji, 1962; Tsuji and Perkins, 1962; Roberts, 1965, 1967; Strasdine and Melville, 1968; Hawirko et al., 1979). Furthermore, the four different C. botulinum groups have distinct physiology and metabolism and therefore they respond differently to environmental cues (Woods and Jones, 1987) and assumed triggers of sporulation (Roberts, 1967; Solomon and Kautter, 1979). The routine growth media used for C. botulinum Group II strains yield insufficient numbers of spores for downstream applications [e.g., tryptone-peptone-glucose-yeast extract broth (TPGY) $10^{5}$ spores/ml (Mascher et al., 2017)]. Several studies have shown evidence that biphasic media consisting of a solid agar phase and a liquid water phase can support efficient sporulation of Group II strains. However, the solid phase is prone to crumbling and thus makes sampling for many downstream applications difficult (Bruch et al., 1968; Peck et al., 1992). In order to facilitate spore biology research in C. botulinum Group II, a medium triggering efficient sporulation and enabling easy sampling is urgently needed.

Here we took the opportunity to exemplify CRISPR-Cas9 "bookmark" technology, a recently proposed concept (Seys et al., 2020) for gold standard complementation studies, which exploits CRISPR-Cas9 to replace the mutant allele in situ with a wild type (WT) allele. Moreover, we established a novel medium for the characterization of spore formation in C. botulinum Group II strains and used it to determine the suitability of a previously described CRISPR-Cas9 system (Ingle et al., 2019) for spore mutant generation in these strains. To exemplify these systems, we targeted the spo0A gene of C. botulinum Group II strain Beluga as its inactivation has been shown to result in an asporogenous phenotype (Al-Hinai et al., 2015; Mascher et al., 2017). The exemplified procedures form a solid basis for future spore-related studies in C. botulinum Group II strains.

\section{MATERIALS AND METHODS}

\section{Experimental Design}

The study consisted of four parts: (1) sporulation mutants of C. botulinum Group II type E strain Beluga were constructed using a novel CRISPR-Cas9 bookmark approach (Seys et al., 2020). 
(2) Growth and sporulation of WT Beluga was tested in seven different biphasic media. The spore and viable cell counts were enumerated directly after inoculating the medium, and subsequently at 1 and 6 days after inoculation. (3) Growth and sporulation of three different Group II strains (Beluga, Eklund 17B, FT10F) were tested in cooked meat medium-TPGY (CMMTPGY), which was the medium selected as best meeting the study criteria in (2), and in two control media: TPGY and Duncan-Strong (DS), the latter supporting the sporulation of Clostridium perfringens (Duncan and Strong, 1968). Spore and viable cell counts were enumerated directly after inoculating the medium, and subsequently at 1 day, 1 week, and 2 weeks after inoculation. Phase-contrast microscopy of the cultures was performed 2 weeks after inoculation. (4) The mutant strains constructed in (1) were characterized and validated in CMM-TPGY. Spore and viable cell count assays and phase-contrast microscopy were performed as in (3).

\section{Strains, Media, and Growth Conditions}

All the C. botulinum and Escherichia coli strains used are listed in Supplementary Table S1. C. botulinum cultures were routinely grown at $30^{\circ} \mathrm{C}$ in an anaerobic workstation (MG1000 anaerobic workstation; Don Whitley Scientific Ltd., Shipley, United Kingdom) with an atmosphere of $85 \% \mathrm{~N}_{2}, 10 \% \mathrm{CO}_{2}$ and $5 \% \mathrm{H}_{2}$. Spore or glycerol stocks of C. botulinum strains were revived in TPGY broth composed of $5 \%(\mathrm{w} / \mathrm{v})$ tryptone, $0.5 \%$ peptone, $2 \%$ yeast extract (Difco, BD Diagnostic Systems, Sparks, MD), 0.4\% glucose (VWR Chemicals, Leuven, Belgium), and $0.1 \%$ sodium thioglycolate (Merck, Darmstadt, Germany) by inoculating $5 \mathrm{ml}$ of the medium with either $5 \mu \mathrm{l}$ of spore stock or $50 \mu \mathrm{l}$ of glycerol stock and incubating until growth reached stationary phase. An overnight culture was prepared by inoculating $5 \mathrm{ml}$ of TPGY with $50 \mu \mathrm{l}$ of revived culture. All the media used for C. botulinum growth experiments were deoxygenated prior to use, either by boiling for $20 \mathrm{~min}$ or by exposure to anaerobic conditions for at least $24 \mathrm{~h}$. All tests were run in triplicate.

In part 1 of the study, transformed strains were grown on TPGY plates containing $1.5 \%(\mathrm{w} / \mathrm{v})$ bacteriological agar supplemented with $250 \mu \mathrm{g} \mathrm{ml}^{-1}$ cycloserine (Merck) and $15 \mu \mathrm{g} \mathrm{ml}^{-1}$ thiamphenicol (Merck). E. coli strains used for cloning and conjugation procedures were grown in LuriaBertani (LB; Invitrogen, Carlsbad, CA) broth or on LB agar plates incubated aerobically at $37^{\circ} \mathrm{C}$. For molecular cloning, LB was supplemented with $25 \mu \mathrm{g} \mathrm{ml}^{-1}$ chloramphenicol (Merck) and for conjugation with $25 \mu \mathrm{g} \mathrm{ml}^{-1}$ chloramphenicol and $30 \mu \mathrm{g} \mathrm{ml}^{-1}$ kanamycin (Merck).

In part 2 of the study, $C$. botulinum strain Beluga was grown in seven different biphasic media: (i) egg-yolk-agar (EYA) $-\mathrm{H}_{2} \mathrm{O}$ consisting of a solid EYA phase [2\% peptone, $0.5 \%$ yeast extract, $0.5 \%$ tryptone, $0.5 \% \mathrm{NaCl}, 10 \%(\mathrm{v} / \mathrm{v})$ egg yolk emulsion (Oxoid Microbiology Products, Basingstoke, United Kingdom), and 1.5\% agar] and a liquid phase of sterile water; (ii) TPGY- $\mathrm{H}_{2} \mathrm{O}$ consisting of a solid TPGY agar phase (TPGY broth with $1.5 \%$ bacteriological agar (Amresco, Ohio, United States) and a liquid phase of sterile water; (iii) Reinforced
Clostridial Medium (RCM) $-\mathrm{H}_{2} \mathrm{O}$ consisting of a solid RCM phase [Reinforced Clostridial Medium (Lab M limited, Lancashire, United Kingdom) prepared according to manufacturer's instructions, and $1.5 \%$ bacteriological agar] and a liquid phase of sterile water; (iv) $\mathrm{CMM}$ ) $-\mathrm{H}_{2} \mathrm{O}$ consisting of a solid phase of $10 \%(\mathrm{w} / \mathrm{v})$ cooked meat medium (Oxoid) with $0.1 \%$ glucose and $1.5 \%$ agar, and sterile water; (v) CMM-TPGY consisting of a solid CMM phase as in (iv) and a liquid phase of TPGY broth; (vi) agar-TPGY consisting of a solid phase of $1.5 \%$ agar and a liquid phase of TPGY broth, and (vii) agar- $\mathrm{H}_{2} \mathrm{O}$ consisting of a solid phase of $1.5 \%$ agar and a liquid phase of sterile water. All biphasic media used in part 2 had a $150-\mathrm{ml}$ solid phase and a $20-\mathrm{ml}$ liquid phase. The liquid phase was inoculated with $5 \mathrm{ml}$ of overnight culture in TPGY to a total volume of $25 \mathrm{ml}$.

In part 3 of the study, Group II strains Beluga, Eklund $17 \mathrm{~B}$, and FT10F were grown in CMM-TPGY containing a solid phase of $75 \mathrm{ml}$ and a $50 \mathrm{ml}$ liquid phase. TPGY broth and DS medium [composed of Modified Duncan-Strong Medium (HiMedia Laboratories Pvt. Ltd., Mumbai, India] prepared according to the manufacturer's instructions, volume $75 \mathrm{ml}$ ) were used as controls. The media were inoculated with $75 \mu \mathrm{l}$ of overnight culture in TPGY.

In part 4 of the study, Beluga sporulation mutant strains, a plasmid control strain, and the WT strain were grown in CMM-TPGY supplemented with $15 \mu \mathrm{g} \mathrm{ml}^{-1}$ thiamphenicol when appropriate.

\section{Plasmid Design and Mutant Construction}

In part 1 of the study, we constructed sporulation mutants of C. botulinum Beluga using a novel CRISPR-Cas9 bookmark approach (Seys et al., 2020). All PCR reactions for the cloning procedures were performed using the KOD Hot-Start high fidelity DNA polymerase (Merck). For DNA purification, the GeneJET PCR Purification Kit (Thermo Fisher Scientific, Waltham, MA) or Monarch DNA Gel Extraction Kit (New England Biolabs, Ipswich, MA) was used. Ligation reactions were performed using T4 DNA ligase (Promega Corporation, Madison, WI). For the restriction digestion of DNA, NEB restriction endonucleases were used (New England Biolabs). Plasmid extractions were carried out using the GeneJET Plasmid Miniprep Kit (Thermo Fisher Scientific). Colony PCR screening was performed using the Phusion high-fidelity DNA polymerase (Thermo Fisher Scientific). The DNA concentration was measured using the NanoDrop 1,000 Spectrophotometer (Thermo Fisher Scientific). For DNA size reference in gel electrophoresis, $1 \mathrm{~kb}$ Plus DNA Ladder was used (New England Biolabs).

All the primers (Metabion International AG) used are listed in Supplementary Table S2. To construct the pMTL431511Beluga $\Delta$ spo0A::bm plasmid for generating a $\Delta$ spo0A::bm genome alteration, we followed a two-step cloning procedure. Firstly, we constructed CRISPR-Cas9 plasmid pMTL431511-Beluga $\triangle$ spo0A, which served as a template for the final pMTL431511Beluga $\triangle$ spo0A::bm plasmid, and carried a copy of a gene encoding truncated but functional Cas9 (Ingle et al., 2019). For the construction of pMTL431511-Beluga $\triangle$ spo0A, 
we generated a knockout $(\mathrm{KO})$ cassette consisting of fused, 1,000 -bp long regions flanking spo0A in the genome, hereafter called the left and right homology arms (LHA and RHA, respectively). The $\mathrm{KO}$ cassette was designed to remove the genomic spo0A codons 3-270, inclusively, generating an in-frame deletion of spo0A. Fragments encoding LHA and RHA were amplified from the genomic DNA of C. botulinum Beluga WT strain using two primer pairs (i) F _LHA_spo0A-AsiSI with R_LHA_spo0A and (ii) F_RHA_spo0A with R_RHA_spo0AAscI. Obtained PCR products were purified and fused in splicing by overhang extension-polymerase chain reaction (SOE-PCR) using a pair of primers F_LHA_spo0A-AsiSI and R_RHA_spoOAAscI. The resulting DNA fragment contained a complete 2,000-bp spo0A KO cassette, flanked by AsiSI and AscI restriction sites. The 20-nt long DNA sequence encoding sgRNA which redirects Cas9 toward spo0A, was designed using the CRISPR-Cas9 guide design tool available online in the Benchling platform ${ }^{1}$ with default settings. The pMTL431511-compatible DNA fragment containing template for sgRNA was generated in PCR reaction using the universal 95-nt primer R_sgRNA-AsiSI and customized 107-nt primer F_spo0A_sgRNA-SalI which harbored a previously designed template for the sgRNA. The utilized oligonucleotides carried 30-bp overhangs, which enabled their mutual hybridization and thus further nucleotide incorporation to the remaining ssDNA sequence in a PCR reaction. The resulting 172-bp product encoded spoOA-specific sgRNA sequence (5'-CATGCTATAGAAGTAGCGTG-3') fused to Cas9-recognized RNA scaffold flanked by restriction sites SalI and AsiSI compatible with the pMTL431511 modular shuttle vector. pMTL431511 linearized with AscI and SalI was ligated with the digested KO cassette and sgRNA-containing fragment in a ratio of 1:3:3, respectively. Chemically competent NEB 5-alpha E. coli cells were transformed with the ligation mixture, plated on selective solid LB medium, and incubated overnight at $37^{\circ} \mathrm{C}$. Resulting antibiotic-resistant colonies were screened in colony PCR with the primer pair F_Pthl_scr and 83XXX-LR, which flank the insert-containing plasmid region. Positive colonies were incubated overnight in $5 \mathrm{ml}$ of selective LB broth at $37^{\circ} \mathrm{C}$ with shaking. The plasmid pMTL431511-Beluga $\triangle$ spooA was extracted from E. coli cultures and sequenced using primer pair F_spo0A_seq and $R \_s p o 0 A \_s e q$ in Sanger sequencing to confirm the correct insert sequence.

Vector pMTL431511-Beluga $\Delta$ spo0A::bm was constructed by inserting 24-bp bookmark sequence into the LHA and RHA junction localized on pMTL431511-Beluga $\Delta s p o 0 A$. The bookmark sequence was designed to contain an efficient 20-nt Cas9-targeting sequence, 3-nt Protospacer Adjacent Motif (PAM), and an additional adenine to maintain the gene alteration in-frame. Shuttle vector pMTL431511-Beluga $\triangle$ spoOA was spliced into two fragments in PCR amplification: the first fragment was generated using the F_spo0A_bm and R_cas primers, and the second with F_cas and R_spo0A_bm. Primers F_spo0A_bm and R_spo0A_bm contained complementary overhangs which encoded the bookmark sequence, and were used to generate the insertion into the LHA and RHA junction. The resulting

${ }^{1}$ https://www.benchling.com/ fragments were fused in a NEBuilder reaction (New England Biolabs) following the manufacturer's recommendations. Positive E. coli clones were selected through colony PCR screening using the primer pair F_bm_scr and 83XXX-LR. The obtained plasmid pMTL431511-Beluga $\Delta$ spo0A::bm was sequenced with Sanger sequencing to verify the correct sequence of the bookmark insertion.

Of note, the final CRISPR-Cas9 vector pMTL431511-Beluga $\triangle$ spo0A::bm was generated using a two-step procedure where we utilized a previously constructed pMTL431511-Beluga $\triangle$ spoOA vector available in our laboratory. If no pre-existing vector is available, we would recommend performing a regular one-step cloning.

For the construction of vector pMTL431511-Beluga::spo0A-wm, a 2,804-bp complementation cassette was assembled. The fragments encoding LHA and RHA were amplified from the genomic DNA of C. botulinum Beluga WT strain, using two primer pairs: (i) F_LHA_spo0A-AsiSI with R_spo0A_wm, and (ii) F_spo0A_wm with R_RHA_spo0A-AscI. The resulting DNA fragments were fused in SOE-PCR yielding a full complementation cassette. A sgRNA-encoding bookmarktargeting fragment (5'-GTACGACACCTCGATCACCA-3') was synthesized as described above, using primers F_bm_sgRNASalI and R_sgRNA-AsiSI. Ligation of DNA fragments and E. coli transformation were performed as described earlier. Positive colonies were screened with colony PCR using primers F_spo0A_ seq and R_spo0A_seq. The constructed plasmid pMTL431511Beluga spoOA-wm was sequenced to confirm the correct insert sequence.

The conjugation donor E. coli CA434 (Purdy et al., 2002) was transformed with an appropriate plasmid and grown overnight in liquid selective $\mathrm{LB}$ broth at $37^{\circ} \mathrm{C}$ with shaking. An aliquot of $1 \mathrm{ml}$ of stationary-phase overnight culture was washed with $1 \mathrm{ml}$ of anaerobic phosphate-buffered saline (PBS). The E. coli cell pellet was re-suspended with $200 \mu \mathrm{l}$ of C. botulinum overnight culture in an anaerobic chamber. The cell suspension was spotted on an anaerobic TPGY agar plate and incubated to ensure conjugation for $8-10 \mathrm{~h}$ at $30^{\circ} \mathrm{C}$ in the anaerobic chamber. The bacterial growth was scraped with a sterile inoculation loop and re-suspended in $500 \mu \mathrm{l}$ of anaerobic PBS which was subsequently spread onto three selective TPGY-agar plates. The plates were incubated at $30^{\circ} \mathrm{C}$ for $24 \mathrm{~h}$ in the chamber. The C. botulinum mutant colonies resulting from conjugation of C. botulinum WT strain with E. coli CA434 pMTL431511-Beluga $\Delta$ spo0A::bm were screened through colony PCR using primers F_spo0A_scr and R_spo0A_scr. Positive mutant colonies were plated on non-selective TPGY-agar plates anaerobically for 2 days at $30^{\circ} \mathrm{C}$. Several resulting random colonies were picked and replica-plated on non-selective and selective TPGY agar plates. The clones growing only on non-selective TPGY plates were stored and confirmed by PCR as plasmid-cured. The plasmid loss frequency for C. botulinum Beluga strain is around $50-60 \%$ of screened colonies.

Primers F_spo0A_scr and R_spo0A_seq were used to screen for complemented $C$. botulinum colonies resulting from conjugation of C. botulinum $\triangle$ spo0A::bm with E. coli CA434 pMTL431511-Beluga::spo0A-wm. The PCR products obtained from colony PCR reactions were separated in an agarose 
gel $(1.5 \% \mathrm{w} / \mathrm{v})$ to verify the amplicon size. After initial confirmation of the complementation, the PCR products derived from positive clones were purified and $600 \mathrm{ng}$ of each was digested with PstI-HF (New England Biolabs) for $1 \mathrm{~h}$ at $37^{\circ} \mathrm{C}$. An aliquot of $25 \mu \mathrm{l}$ of the digests were separated in an agarose gel to reveal the resulting digestion pattern. Sanger sequencing confirmed the successful complementation and correct single-nucleotide modifications. CRISPR-Cas9 plasmid was cured from the positive mutant colonies as described above.

\section{Spore and Viable Cell Count Assays}

In parts 2-4 of the study, spore counts were determined using spore heating assays. A $200-\mu$ l aliquot of culture was incubated for $20 \mathrm{~min}$ at $60^{\circ} \mathrm{C}$ to eliminate vegetative cells. Heat-treated samples were serially diluted in fresh TPGY and, whenever applicable, supplemented with $15 \mu \mathrm{g} \mathrm{ml}^{-1}$ thiamphenicol, in 96-well plates and incubated anaerobically for 5 days at $30^{\circ} \mathrm{C}$. The most probable number technique with three tubes was applied to enumerate heat-stable spores (Blodgett, 2000). Non-heated aliquots were similarly investigated to estimate viable cell counts.

\section{Phase-Contrast Microscopy}

Phase-contrast microscopy was used to monitor sporulation 14 days after inoculation in parts 3 and 4 of the study. The cultures were gently mixed and $200-\mu$ l culture aliquots were centrifuged for $2 \mathrm{~min}$ at $5000 \times g$, the supernatant was discarded, and cell pellet was re-suspended in $30-50 \mu \mathrm{l}$ of anaerobic PBS. A small amount $(2 \mu \mathrm{l})$ of resulting cell suspension was immobilized on a thin layer of $1.7 \%$ agarose (SeaKem LE agarose, Lonza) coated on the surface of a microscopy slide. Cells were visualized in DMi8 Leica inverted fluorescent microscope equipped with HC PL APO 100x/1.40 OIL PH3 objective and Hamamatsu Orca Flash V2 LT camera. Microscopy images were captured using the Metamorph Basic Acquisition for Microscope software and adjusted and analyzed with COREL PaintShop Pro x9 and Fiji ImageJ version 1.51, respectively.

\section{Statistical Analysis}

One-way ANOVA was used to compare the total viable cell and spore counts of each strain in different media at different time points in part 3 of the study. Student's $t$-test was used to compare total viable cell counts or spore counts of mutant and complemented strains to the WT or respective plasmid control strain in part 4 of the study.

\section{RESULTS AND DISCUSSION}

\section{Construction of $C$. botulinum Group II Mutants Using CRISPR-Cas9 Bookmark Approach}

The assignment of function to a particular gene product requires that the phenotypic properties of a mutant culture in which the encoding gene has been deleted are compared to those of the progenitor, WT culture. However, the phenotype of the isolated mutant may be a consequence of the acquisition of ancillary mutations elsewhere in the genome during the mutagenesis procedure, which may be responsible for the changed phenotype. To assign the observed phenotype to the absence of the deleted gene, it is essential that a complementation test is performed in which a functional copy of the deleted gene is introduced back into the mutant cells and the phenotype of the complemented mutant shown to be restored to that of the progenitor, WT cell. As the complementation method itself can also cause unintended changes in phenotype, especially if the introduced gene is on an autonomous plasmid, we chose to experimentally validate a recently proposed concept of bookmark complementation (Seys et al., 2020). In essence, the procedure restores the mutant to the WT configuration by replacing the mutant allele in the chromosome with the WT gene. It relies on prior incorporation of a unique 24-nucleotide (nt) bookmark sequence into the mutant allele that acts as a single guide RNA target for its subsequent Cas9-mediated substitution with the WT allele.

As the first practical demonstration of the bookmark concept (Seys et al., 2020), we chose to target the spo0A of our model C. botulinum Group II strain Beluga. Accordingly, we used the previously described (Seys et al., 2020) KO system, based on the CRISPR-Cas9 of Streptococcus pyogenes, to delete spo0A and replace it with a mutant allele comprising a 24-nt bookmark sequence flanked by the first and last two codons of the structural gene. The bookmark (Figure 1) was designed by applying a GC-rich sequence comprised an efficiently cleaved 20-nt CRISPR-Cas9 target (efficiency score calculated using algorithm available on https://www.benchling.com/), 3-bp PAM and an additional base pair maintaining the resulting gene deletion in frame. An nBLAST analysis of the bookmark sequence against the order Clostridiales indicated no matches in the genome sequences currently available in public databases, suggesting it could find wide use in these bacteria. By applying this approach, we generated a $C$. botulinum Beluga $\triangle$ spo0A::bm deletion mutant. Following the transfer of the CRISPR-Cas9 deletion vector, we performed colony PCR screening of 12 randomly picked antibiotic-resistant colonies. Six out of the twelve colonies screened were deletion mutants, and thus the

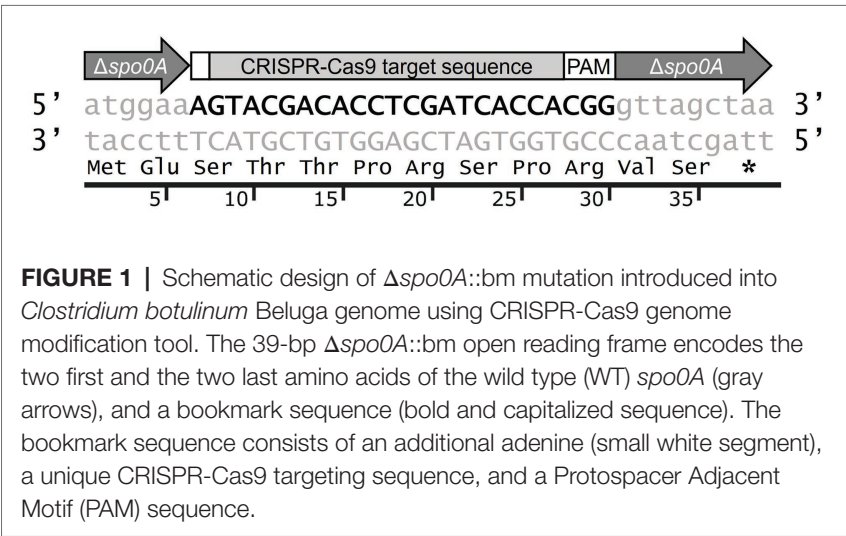


mutant generation success was 50\% (Supplementary Figure S1). CRISPR-Cas9 deletion vector was cured from the mutant strain and Sanger sequencing confirmed the expected sequence of the $\Delta$ spo0A::bm modification (Supplementary Figure S2).

To demonstrate the applicability of the gene bookmarking approach in C. botulinum, we restored a functional spo0A copy into the original chromosomal locus by redirecting the CRISPR-Cas9 machinery toward the introduced bookmark sequence in the $\triangle$ spo0A::bm mutant. The functional copy of spo0A was not the native gene as this would result in a complemented strain that would be indistinguishable from the progenitor WT cell. Consequently, contamination of a culture with the WT strain would be difficult to rule out (Seys et al., 2020). Instead, the gene used for complementation should contain a "watermark" sequence that would distinguish the complemented clones from the WT (Seys et al., 2020). Importantly, the nucleotide changes made should not cause a change to the encoded protein. The applied approach is to make changes that either create, or remove, a restriction enzyme recognition sequence. This allows the authenticity of the clone to be rapidly established through restriction digestion of an amplified PCR fragment encompassing the region in question ( $\mathrm{Ng}$ et al., 2013). Here we chose to introduce a watermark sequence, comprising silent changes to five separate codons $(267 \mathrm{~A}>\mathrm{T}, 270 \mathrm{~A}>\mathrm{T}, 273 \mathrm{~T}>\mathrm{A}, 285 \mathrm{~T}>\mathrm{A}, 288 \mathrm{~A}>\mathrm{T})$ in the spo0A gene (Supplementary Figure S5). When designing the watermark sequence, we considered the codon usage of C. botulinum species by replacing the WT codons with similarly used synonymous ones. We retrieved the codon usage table for C. botulinum from online codon usage database (Nakamura et al., 2000). For the watermark sequence we used codons with similar usage to the ones originally found in the WT strain applying the following changes: codon GCA to GCT (frequency 0.47 and 0.44), GTA to GTT (frequency 0.49 and 0.42), GGT to GGA (frequency 0.34 and 0.5 ), ATT to ATA (frequency 0.40-0.54), and ACA to ACT (frequency 0.45-0.46).

The watermark lacks the PstI-recognized nucleotide sequence found within the original spoOA sequence. Altogether, the introduction of a watermark aims at leaving a genomic imprint in the restored gene copy and facilitates the distinction of complemented from the wild type strain by revealing different digestion patterns of the colony PCR generated DNA fragments. Following transfer of the requisite CRISPR-Cas9 complementation vector into the $\Delta$ spo0A::bm mutant, 12 antibiotic-resistant transconjugants were selected and all (100\%) were shown by colony PCR (Supplementary Figure S3) and PstI digestion (Supplementary Figure S4) to be complemented watermarkharboring strains. Sanger sequencing of a random selection of clones confirmed that the expected region of the DNA had been inserted (Supplementary Figure S5). The watermark sequence applied in this study removes an already existing restriction enzyme site. However, this approach might not be readily applicable due to the limited restriction enzyme availability. In such case, the watermark-specific screening could be performed through PCR. One of the primers applied for the amplification should directly anneal to the watermarked genomic sequence with altered nucleotides at the 3 ' end, which would increase the specificity of the primer. That way, the watermark-specific primer, together with another regular primer annealing nearby, would yield a PCR product only in the case of successful watermark insertion. However, we would recommend validating the watermark-specific primer before the actual mutant screening to ensure the specificity of primer and expected amplification result.

The successful creation of C. botulinum $\triangle$ spo0A::bm and $\triangle$ spo0A::bm::spo0A-wm strains served to illustrate how the utility of the bookmark approach transcends simple complementation and can be employed to deliver derivatized genes to the genome. In this case, the modified gene contained five silent nucleotide changes, but could equally be applied to incorporate alterations in encoded amino acids for the purposes of structural or mechanistic studies. Successful complementation of $s p o 0 A$ harboring the pre-designed nucleotide changes provides a foundation for utilizing the bookmark approach to introduce other genomic alterations within a copy of a restored gene, i.e., modifications of putative protein binding boxes, singleamino-acid substitutions or deletions, or construction of reporter gene fusions.

\section{Selection of Efficient Sporulation Media for C. botulinum Group II}

An integral part of spore research is the availability of a laboratory medium that ensures sufficient and reproducible spore yields and which enables downstream processing. Roberts (1967) identified three different media likely support clostridial sporulation: (i) cooked meat medium; (ii) blood agar; and (iii) TPAY-GT composed of tryptone, peptone, ammonium sulfate, yeast extract, glucose, and sodium thioglycolate. No single medium yielded satisfactory results for all tested $C$. botulinum strains (Roberts, 1967). TPGY, a variation of TPAY-GT, eventually became the routine growth medium for C. botulinum, and also provided a functional sporulation medium for Group I strains (Eklund et al., 1969). However, while effectively supporting growth, TPGY is not an ideal sporulation medium for Group II strains (Mascher et al., 2017).

Different variations of cooked meat medium have been used to sporulate C. botulinum (Perkins, 1965; Roberts, 1967; Solomon and Kautter, 1979), despite difficulties in downstream processes caused by small meat particles floating in the medium (Perkins, 1965; Roberts, 1967). Peck (1992) used a biphasic sporulation medium consisting of a solid phase of CMM supplemented with agar and glucose, and a liquid phase of sterile water, to collect Group II spores for purification and heat inactivation assays (Peck et al., 1992). Other biphasic media with different solid phases and a liquid phase of sterile water have also been shown to support sporulation of Group II strains (Perkins, 1965; Bruch et al., 1968; Peck et al., 1992). The mechanism of a biphasic medium supporting efficient sporulation remains unclear, but it is possible that attachment to a solid surface induces sporulation (Vlamakis et al., 2008). Liquid phases of nutrient-rich broths likely support growth better than a water phase, and thus we expected nutrient-rich liquid phases primarily 
to yield a larger number of sporulation-prone cells than water (Peck et al., 1992; Braconnier et al., 2001; Brunt et al., 2018).

Our goal was to find a C. botulinum Group II sporulation medium that would be straightforward to prepare, support efficient growth and sporulation, and enable easy and particlefree sampling. Therefore, we first screened the growth and sporulation of C. botulinum Group II strain Beluga in five different biphasic media: TPGY- $\mathrm{H}_{2} \mathrm{O}$, EYA- $\mathrm{H}_{2} \mathrm{O}, \mathrm{RCM}-\mathrm{H}_{2} \mathrm{O}$, CMM- $\mathrm{H}_{2} \mathrm{O}$, and CMM-TPGY. Agar-TPGY and agar- $\mathrm{H}_{2} \mathrm{O}$ were used as controls. All media except for agar- $\mathrm{H}_{2} \mathrm{O}$ supported the growth and sporulation of Beluga, with concentrations of approximately $10^{7}-10^{8}$ cells or spores $/ \mathrm{ml}$ being reached in all of them (Supplementary Figure S6). However, the solid phase of TPGY- $\mathrm{H}_{2} \mathrm{O}$, EYA- $\mathrm{H}_{2} \mathrm{O}$, and $\mathrm{RCM}-\mathrm{H}_{2} \mathrm{O}$ was prone to crumbling, causing pipette tips to become clogged by small agar pieces during sampling and considerably interfered with downstream processes, preventing the collection of spores by centrifugation. CMM- $\mathrm{H}_{2} \mathrm{O}, \mathrm{CMM}-\mathrm{TPGY}$, and agar-TPGY all enabled effortless sampling, and CMM-TPGY resulted in better growth and subsequently higher spore concentration than the other two media (approximately $1 \log$, Supplementary Figure S6). This indicates that nutrient-rich solid and liquid phases support growth better than poorer media and, as a result, enable higher spore yields, even if there are no differences in the sporulation rate (maximum spore count divided by the maximum total cell count). Fulfilling our criteria better than the other media, CMM-TPGY was selected for further validation.

To evaluate the suitability of CMM-TPGY as a sporulation medium for different Group II C. botulinum strains, we used it to culture Beluga (toxin type E1), Eklund 17B (toxin type B4) and FT10F (toxin type F6) for 14 days at $30^{\circ} \mathrm{C}$ under anaerobic conditions. The standard TPGY broth (Solomon and Lilly, 2001) and DS used to sporulate C. perfringens (Duncan and Strong, 1968) were used as controls. We collected samples for viable cell and spore enumeration immediately after inoculation, day 1 , day 7 , and day 14 . At the end of the growth period, we performed phase-contrast microscopy of the cultures. All three strains reached their maximum total viable cell counts 1 day after inoculation in all different media. The growth of Beluga and FT10F was best supported by TPGY broth and CMM-TPGY, with significantly lower $(p<0.05)$ total cell count detected in DS broth (Figure 2A). The growth of Eklund 17B was best supported by CMM-TPGY, with significantly lower total cell counts reached in TPGY and DS broths (Figure 2A). Although TPGY broth supported vegetative growth (Figure 2A, day 1), especially in case of strains Beluga and FT10F, sporulation in TPGY was generally poor. All three Group II strains reached significantly higher $(p<0.05)$ spore concentrations in the biphasic CMM-TPGY medium than in TPGY broth alone on days 1 , 7, and 14 (Figure 2B). The sporulation rates were similar in CMM-TPGY and DS broths for all strains, but due to higher total cell counts, the maximum spore concentrations reached in CMM-TPGY were significantly higher $(p<0.05)$ than those in DS. The results indicate that abundant growth leading to a concentrated culture (Tyrrell et al., 1958) in combination with potential factors supporting sporulation, such as attachment
(Vlamakis et al., 2008), biofilm formation (Branda et al., 2001), or sporulation-activating cell-to-cell communication due to cell crowding (Cooksley et al., 2010; Li et al., 2011) are prerequisites for a high spore yield.

Phase-contrast microscopy of Beluga, Eklund 17B and FT10F grown in CMM-TPGY, TPGY, and DS (Figure 3) supported the results of spore heating assays. Cultures grown in CMM-TPGY showed an abundance of phase-bright mature spores, as opposed to vast amounts of vegetative cell debris and only few spores observed in TPGY, and little cell debris and few spores observed in DS.

\section{Characterization of Constructed CRISPR-Cas9 Mutants in CMM-TPGY Medium}

To characterize the phenotype of the spo0A mutants, we performed a comprehensive sporulation test utilizing the biphasic CMM-TPGY medium for cultivating C. botulinum Group II Beluga strains $\triangle$ spo0A::bm and $\triangle$ spo0A::bm::spo0A-wm. Spo0A functions as a master regulator of sporulation in all spore-forming bacteria (Hoch, 1993; Al-Hinai et al., 2015), including C. botulinum Group II (Mascher et al., 2017). We therefore expected the spo0A deletion to prevent the cells from entering sporulation. Since spore formation can be readily traced in the laboratory, an asporogenous phenotype would serve as a reliable control for successful gene knock-out and for in cis or in trans complementation of the mutations. To compare the efficiency of a chromosomal gene complementation to the conventional plasmid complementation approach, we constructed a C. botulinum Beluga $\Delta$ spo0A::bm-pMTL82151::spo0A strain harboring shuttle vector with a WT copy of spo0A under the control of its native promoter (Mascher et al., 2017).

To provide suitable control strains capable of growing in a selective medium, along with the $\triangle$ spo0A::bmpMTL82151::spo0A strain, we also characterized the WT-pMTL82151 and $\Delta$ spo0A::bm-pMTL82151 control strains, both carrying just the pMTL82151 shuttle vector. All the strains were cultivated for 2 weeks in CMM-TPGY medium in three biological replicates. The plasmid-carrying cultures were supplemented with $15 \mu \mathrm{g} \mathrm{ml}^{-1}$ thiamphenicol every $48 \mathrm{~h}$ to maintain sufficient concentration of antibiotic during the extended incubation.

As expected, the mutant $\Delta s p o 0 A:$ bm was unable to enter sporulation, which was verified by the absence of heat-resistant spores (Figure 4B) and the visual absence of sporulating cells or free spores when subjected to microscopic examination (Figure 5). The maximum average cell count reached in the mutant cultures, $2.54 \times 10^{8}$ cells $/ \mathrm{ml}$, was slightly but significantly $(p=0.039)$ lower than that of the WT strain $\left(1.78 \times 10^{9}\right.$ cells $\left./ \mathrm{ml}\right)$. As the applied total cell count assay enumerates the sum of vegetative cells and spores, a lower concentration of cells in the $\Delta$ spo0A::bm culture is likely due to lack of spores. The sporulating phenotype was successfully restored by returning a watermark-harboring $s p o 0 A$ copy in the original spoOA locus applying the CRISPR-Cas9 bookmark-targeting approach. The complemented $\triangle$ spo0A::bm::spo0A-wm mutant showed no 
A
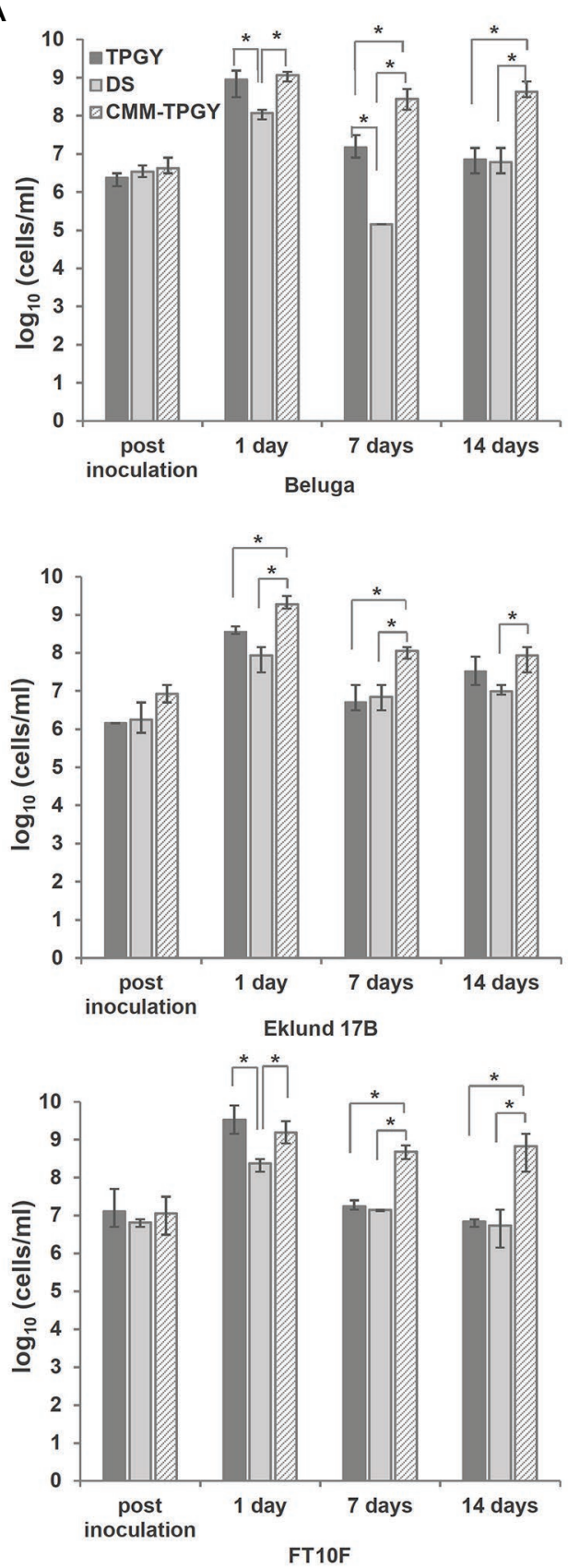

B
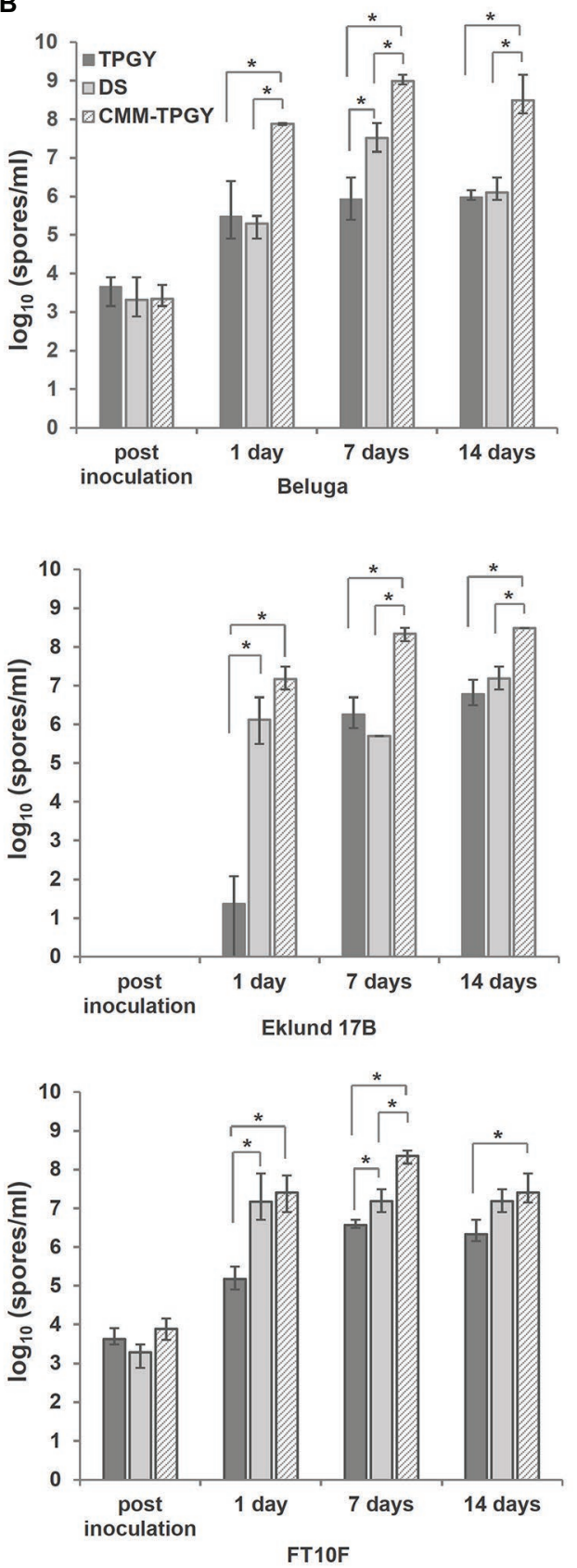

FIGURE 2 | Comparison of viable cell and spore counts produced by three different C. botulinum Group II strains (Beluga, Eklund 17B and FT10F) grown in three challenged media: standard tryptone-peptone-glucose-yeast extract (TPGY) broth, Clostridium perfringens sporulation medium Duncan-Strong (DS), and the newly described biphasic cooked meat medium-TPGY (CMM-TPGY) broth. Total viable cell and spore enumeration (A) and spore heating assays (B) were performed directly after inoculating the fresh media, and subsequently at day 1, day 7, and day 14 post inoculation. Biphasic CMM-TPGY medium promoted most efficiently bacterial growth (A) and sporulation (B) of all three tested C. botulinum Group II strains. The experiment was performed in three parallel biological replicates. The error bars represent the maximum and minimum values of three replicates. ${ }^{*}$ Statistically significant $(p<0.05)$ difference in cell or spore concentration between different media.

significant differences to WT in the maximum cell concentrations, but the maximum spore counts were approximately 1 log lower in the complemented strains when compared to WT $(p<0.001$; Figure 4A). The difference in sporulation rate between WT and complemented $\triangle$ spo0A::bm::spo0A-wm strain could arise from the limitations of the applied MPN enumeration method that is a statistical approach giving only an estimate number of cells in bacterial suspension, and it is known to 


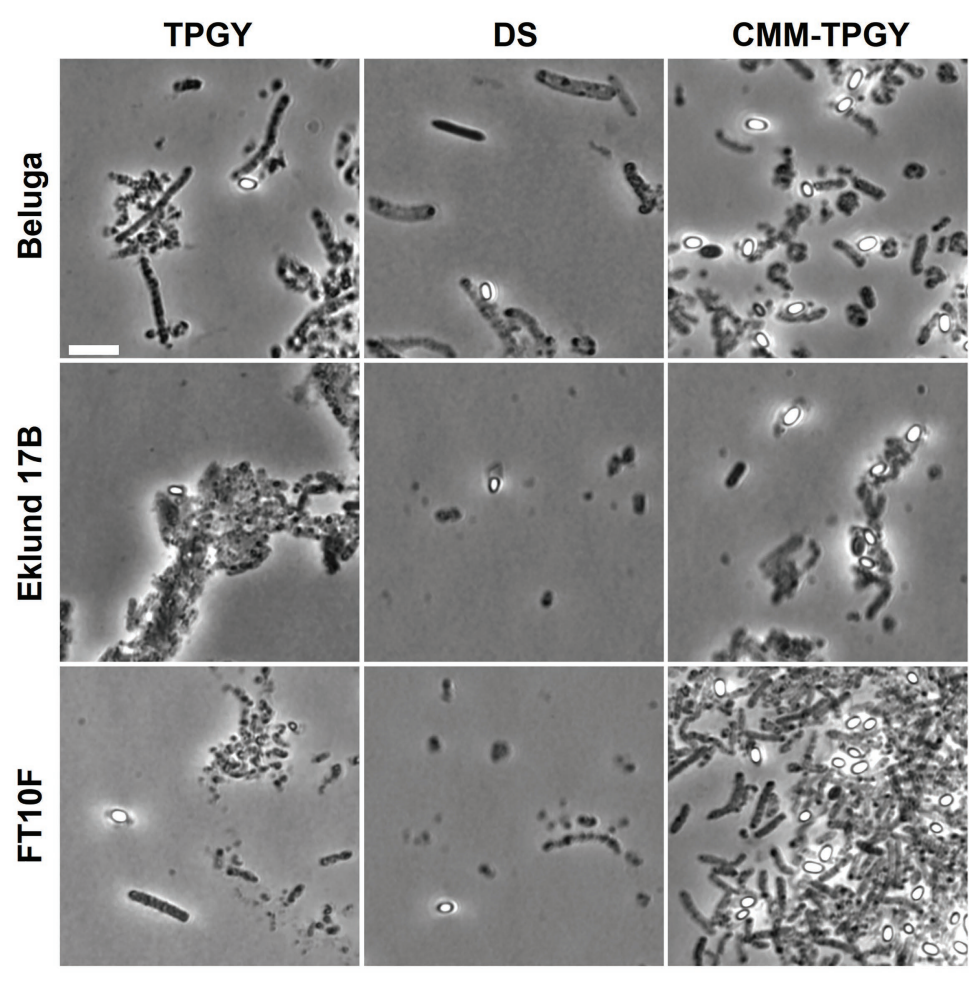

FIGURE 3 | Phase-contrast microscopy of C. botulinum Group II strains Beluga, Eklund 17B and FT1OF after 14 days of growth in TPGY broth, DS broth, and in biphasic CMM-TPGY medium. From the three tested media, the biphasic CMM-TPGY provides the most favorable sporulation conditions for all the tested Group II strains. Scale bar $5 \mu \mathrm{m}$.

A

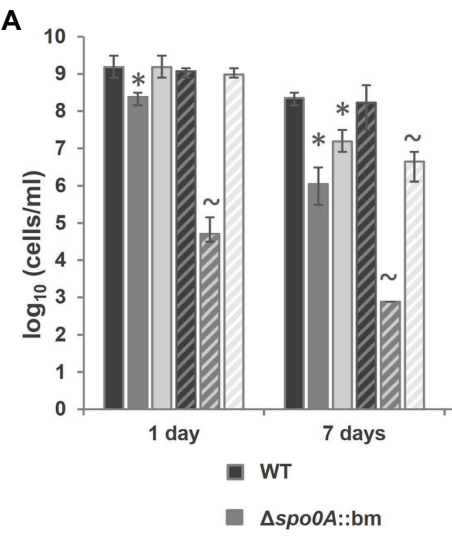

B

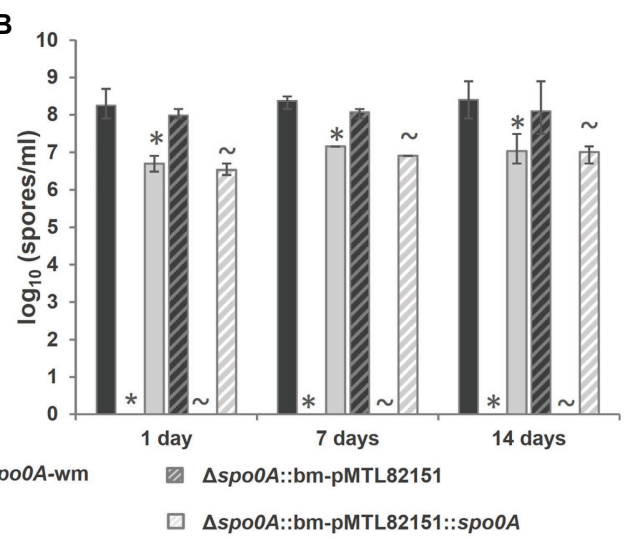

FIGURE 4 | Phenotype characterization in CMM-TPGY sporulation medium of the constructed $\triangle$ spoOA::bm C. botulinum Beluga strain compared to the WT parental and complemented strains, where a copy of functional spoOA-coding sequence was inserted back to the original chromosomal locus

( $\triangle$ spoOA::bm::spo0A-wm) or expressed from a plasmid ( $\triangle$ spo0A::bm-pMTL82151::spoOA). (A) Viable cell and (B) spore quantifications were performed at day 1 , day 7 , and day 14 post inoculation. The $\triangle$ spo0A::bm mutant strain produced significantly smaller total viable cell counts than the WT strain (A) due to the inability to form heat-resistant spores (B). Both complementation strategies allowed comparable restoration of asporogenous phenotype for $\triangle$ spoOA::bm. All the data were obtained from three biological replicates. ${ }^{*}$ Cell or spore concentration of a mutant or complemented strain was significantly $(p<0.05)$ lower than that of WT. ${ }^{-C e l l ~ o r ~}$ spore concentration was significantly $(p<0.05)$ lower than that of the respective plasmid control.

be less accurate in case of dense bacterial populations (Chandrapati and Williams, 2014). Another explanation for the observed difference in spore number would be an effect of secondary SNPs in the genome, presumably caused by stress during the mutational process. Nevertheless, the spores of the complemented strains are produced in relatively high amount; they are heat-resistant and show regular morphology indicating that the entire sporulation pathway is restored and functional. 


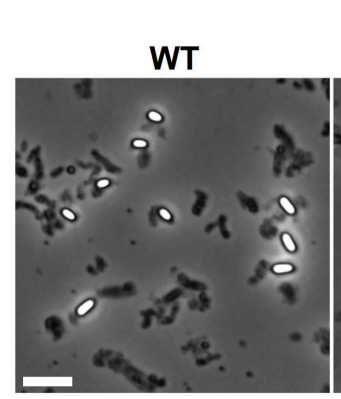

WTPMTL82151

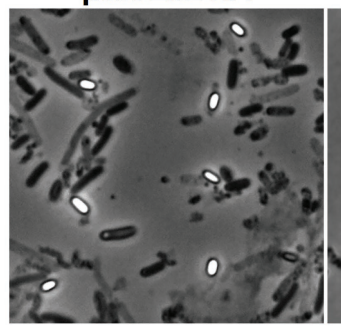

$\triangle$ spooA::bm

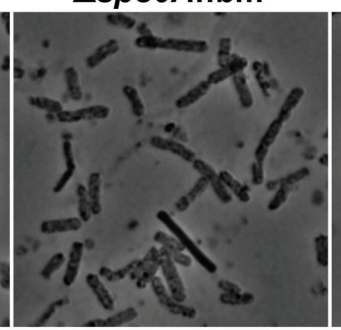

$\triangle$ spo0A::bmPMTL82151

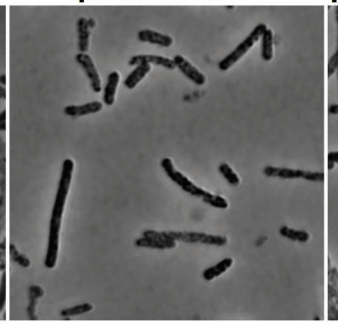

$\triangle$ spo0A::bm ::spo0A-wm

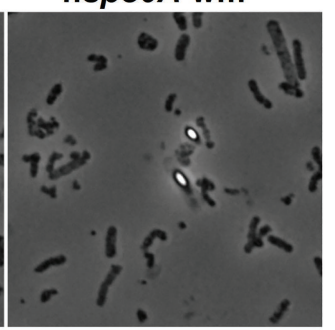

$\triangle$ spo0A::bmpMTL82151::spo0A

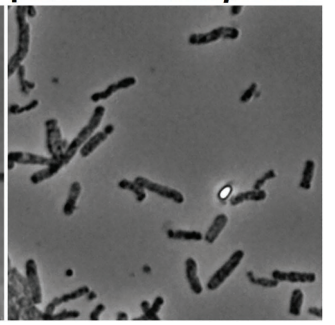

FIGURE 5 | Phase-contrast microscopy images at 14 days post inoculation demonstrating the morphological differences between WT, $\Delta$ spoOA::bm, and complemented mutant strains. Phase-bright endospores were detectable only in samples containing WT or complemented strains. The $\Delta$ spoOA::bm mutant did not display any spores or sporulating cells, suggesting that endospore formation ceased at the early stage. Scale bar $5 \mu \mathrm{m}$.

The plasmid-borne complementation of the spo0A deletion was equally efficient (reaching approximately $10^{7}$ spores $/ \mathrm{ml}$ ) as the $s p o 0 A$-wm insertion into the chromosomal spo0A locus, indicating that the introduction of a bookmark (i) does not interfere with the phenotype, (ii) is unlikely to cause polar effects, and (iii) can be safely used for routine deletiontagging. No differences in growth or sporulation were detected between WT and its plasmid control strain WT-pMTL82151 (Figures 4A,B). However, the second control strain $\Delta$ spo0A::bmpMTL82151 showed significantly lower total cell numbers than the respective mutant $\Delta$ spo0A::bm $(p<0.001)$ and no growth at all on day 14. A possible explanation behind this observation could be a gradual plasmid loss rendering the strain increasingly sensitive to thiamphenicol. In case of the sporulation-deficient strain incubated during extended periods, the control plasmid cannot be preserved inside the spores, therefore, the bacteria lose the ability to grow in antibioticsupplemented broth.

\section{CONCLUSION}

The present study represents the first use of CRISPR-Cas9 in the generation of in-frame deletions in C. botulinum Group II. The system used relies on a truncated Cas9 nickase variant previously only exemplified in Clostridium difficile (Ingle et al., 2019). Using the spo0A gene as the target, we also provided the first practical demonstration of CRISPR-Cas9-mediated bookmark complementation technology (Seys et al., 2020), in which the deleted chromosomally located spo0A gene was restored to a functional copy in situ. This substitution was made possible by the prior incorporation into the mutant allele of a 24-nt bookmark sequence that should be widely applicable in other clostridial species. The functional copy of spo0A used for complementation was "watermarked" with five silent nucleotide changes that removed a WT PstI restriction site but still enabled production of active Spo0A. This was to allow the complemented strain to be easily distinguished from possible contamination with the WT, but also to demonstrate the potential of the bookmark technology in functional studies in which a chromosomal gene is replaced, in a two-step process, with a derivatized copy. In future studies such genes could include, for instance, variants with specific amino acid changes, homologs from other bacterial strains or species, and fusion proteins. Our analysis of the sporulation mutant and the generated complemented strain was made possible by the formulation of a biphasic CMM-TPGY medium, which supported the growth and efficient sporulation of three different C. botulinum Group II strains and enabled easy and particle-free sampling for microscopy and other downstream applications. Altogether, the findings described in this study constitute a solid base for future spore and sporulation studies of C. botulinum Group II strains.

\section{DATA AVAILABILITY STATEMENT}

The original contributions presented in the study are included in the article/Supplementary Material, further inquiries can be directed to the corresponding author. 


\section{AUTHOR CONTRIBUTIONS}

Conceptualization: MBN, AM, GM, and ML. Formal analysis: $\mathrm{MBN}$ and AM. Investigation: $\mathrm{MBN}, \mathrm{AM}, \mathrm{GM}$, and $\mathrm{VH}$. Methodology: MBN, AM, GM, DG, and NPM. Writing the original draft-MBN, AM, and ML. Reviewing and editing the original draft: all authors. All the authors have read and agreed on the published version of the manuscript.

\section{FUNDING}

This research was funded by the European Research Council (ERC-CoG grant no. 683099), Marie Skłodowska-Curie Innovative Training Network CLOSPORE (642068), the Academy of Finland (299700), and by the Doctoral Programme

\section{REFERENCES}

Al-Hinai, M. A., Jones, S. W., and Papoutsakis, E. T. (2015). The Clostridium sporulation programs: diversity and preservation of endospore differentiation. Microbiol. Mol. Biol. Rev. 79, 19-37. doi: 10.1128/MMBR.00025-14

Blodgett, R. (2000). "Bacteriological analytical manual appendix 2: most probable number from serial dilutions" in Bacteriological analytical manual. U.S. Food and Drug Administration.

Braconnier, A., Broussolle, V., Perelle, S., Fach, P., Nguyen-The, C., and Carlin, F. (2001). Screening for Clostridium botulinum type a, B, and E in cooked chilled foods containing vegetables and raw material using polymerase chain reaction and molecular probes. J. Food Prot. 64, 201-207. doi: 10.4315/0362-028X-64.2.201

Branda, S. S., González-Pastor, J. E., Ben-Yehuda, S., Losick, R., and Kolter, R. (2001). Fruiting body formation by Bacillus subtilis. Proc. Natl. Acad. Sci. U. S. A. 98, 11621-11626. doi: 10.1073/pnas. 191384198

Bruch, M. K., Bohrer, C. W., and Denny, C. B. (1968). Adaptation of biphasic culture technique to the sporulation of Clostridium botulinum type E. J. Food Sci. 33, 108-109. doi: 10.1111/j.1365-2621.1968.tb00895.x

Brunt, J., Carter, A. T., Pye, H. V., and Peck, M. W. (2018). The orphan germinant receptor protein GerXAO (but not GerX3b) is essential for L-alanine induced germination in Clostridium botulinum group II. Sci. Rep. 8:7060. doi: 10.1038/s41598-018-25411-x

Cañadas, I. C., Groothuis, D., Zygouropoulou, M., Rodrigues, R., and Minton, N. P. (2019). RiboCas: a universal CRISPR-based editing tool for Clostridium. ACS Synth. Biol. 8, 1379-1390. doi: 10.1021/acssynbio.9b00075

Chandrapati, S., and Williams, M. G. (2014). "Total viable counts: most probable number (MPN)" in Encyclopedia of food microbiology: Second edition. eds. C. A. Batt and M. L. Tortorello (Academic Press), 621-624.

Clauwers, C., and Lood, C., Van Noort, V., and Michiels, C. W. (2017). Canonical germinant receptor is dispensable for spore germination in Clostridium botulinum group II strain NCTC 11219. Sci. Rep. 7:15426. doi: 10.1038/ s41598-017-15839-y

Clauwers, C., Vanoirbeek, K., Delbrassinne, L., and Michiels, C. W. (2016). Construction of nontoxigenic mutants of nonproteolytic Clostridium botulinum NCTC 11219 by insertional mutagenesis and gene replacement. Appl. Environ. Microbiol. 82, 3100-3108. doi: 10.1128/aem.03703-15

Cooksley, C. M., Davis, I. J., Winzer, K., Chan, W. C., Peck, M. W., and Minton, N. P. (2010). Regulation of neurotoxin production and sporulation by a putative agrBD signaling system in proteolytic Clostridium botulinum. Appl. Environ. Microbiol. 76, 4448-4460. doi: 10.1128/AEM.03038-09

Dahlsten, E., Kirk, D., Lindström, M., and Korkeala, H. (2013). Alternative sigma factor SigK has a role in stress tolerance of group I Clostridium botulinum strain ATCC 3502. Appl. Environ. Microbiol. 79, 3867-3869. doi: 10.1128/AEM.04036-12

Duncan, C. L., and Strong, D. H. (1968). Improved medium for sporulation of Clostridium perfringens. Appl. Microbiol. 16, 82-89. in Food Chain and Health of the University of Helsinki and Doctoral Programme in Microbiology and Biotechnology of the University of Helsinki.

\section{ACKNOWLEDGMENTS}

We would like to thank Hanna Korpunen for her excellent technical assistance.

\section{SUPPLEMENTARY MATERIAL}

The Supplementary Material for this article can be found online at: https://www.frontiersin.org/articles/10.3389/fmicb.2021.617269/ full\#supplementary-material

Eklund, M. W., Poysky, F. T., and Boatman, E. S. (1969). Bacteriophages of Clostridium botulinum types a, B, E, and F and nontoxigenic strains resembling type E. J. Virol. 3, 270-274. doi: 10.1128/jvi.3.2.270-274.1969

Grossman, A. D., and Losick, R. (1988). Extracellular control of spore formation in Bacillus subtilis. Proc. Natl. Acad. Sci. U. S. A. 85, 4369-4373. doi: 10.1073/ pnas.85.12.4369

Harris, A. (2015). "Clostridium botulinum" in Encyclopedia of food and health. eds. B. Caballero, P. M. Finglas and F. Toldrá (Academic Press), 141-145.

Hatheway, C. L. (1993). “Clostridium botulinum and other clostridia that produce botulinum neurotoxin" in Clostridium botulinum. Ecology and control in foods. eds. A. Hauschild and K. Dodds (New York: Marcel Dekker), 3-20.

Hawirko, R. Z., Naccarato, C. A., Lee, R. P. W., and Maeba, P. Y. (1979). Outgrowth and sporulation studies on Clostridium botulinum type E: influence of isoleucine. Can. J. Microbiol. 25, 522-527. doi: 10.1139/m79-076

Heap, J. T., Pennington, O. J., Cartman, S. T., Carter, G. P., and Minton, N. P. (2007). The ClosTron: a universal gene knock-out system for the genus Clostridium. J. Microbiol. Methods 70, 452-464. doi: 10.1016/j.mimet.2007.05.021

Hoch, J. A. (1993). Regulation of the phosphorelay and the initiation of sporulation in Bacillus subtilis. Annu. Rev. Microbiol. 47, 441-465. doi: 10.1146/annurev.mi.47.100193.002301

Ingle, P., Groothuis, D., Rowe, P., Huang, H., Cockayne, A., Kuehne, S. A., et al. (2019). Generation of a fully erythromycin-sensitive strain of Clostridioides difficile using a novel CRISPR-Cas9 genome editing system. Sci. Rep. 9:8123. doi: 10.1038/s41598-019-44458-y

Kirk, D. G., Zhang, Z., Korkeala, H., and Lindström, M. (2014). Alternative sigma factors SigF, SigE, and SigG are essential for sporulation in Clostridium botulinum ATCC 3502. Appl. Environ. Microbiol. 80, 5141-5150. doi: 10.1128/ AEM.01015-14

Kuehne, S. A., Rood, J. I., and Lyras, D. (2019). Clostridial genetics: genetic manipulation of the pathogenic clostridia. Microbiol. Spectr. 7. doi: 10.1128/ microbiolspec.gpp3-0040-2018

Li, J., Chen, J., Vidal, J. E., and McClane, B. A. (2011). The Agr-like quorumsensing system regulates sporulation and production of enterotoxin and beta2 toxin by Clostridium perfringens type a non-food-borne human gastrointestinal disease strain F5603. Infect. Immun. 79, 2451-2459. doi: 10.1128/IAI.00169-11

Lindström, M., Kiviniemi, K., and Korkeala, H. (2006). Hazard and control of group II (non-proteolytic) Clostridium botulinum in modern food processing. Int. J. Food Microbiol. 108, 92-104. doi: 10.1016/j.ijfoodmicro.2005.11.003

Mascher, G., Derman, Y., Kirk, D. G., Palonen, E., Lindström, M., and Korkeala, H. (2014). The CLO3403/CLO3404 two-component system of Clostridium botulinum E1 Beluga is important for cold shock response and growth at low temperatures. Appl. Environ. Microbiol. 80, 399-407. doi: 10.1128/AEM.03204-13

Mascher, G., Mertaoja, A., Korkeala, H., and Lindström, M. (2017). Neurotoxin synthesis is positively regulated by the sporulation transcription factor Spo0A in Clostridium botulinum type E. Environ. Microbiol. 19, 4287-4300. doi: $10.1111 / 1462-2920.13892$ 
McAllister, K. N., and Sorg, J. A. (2019). CRISPR genome editing systems in the genus Clostridium: a timely advancement. J. Bacteriol. 201, e00219-e00319. doi: 10.1128/JB.00219-19

Minton, N. P., Ehsaan, M., Humphreys, C. M., Little, G. T., Baker, J., Henstra, A. M., et al. (2016). A roadmap for gene system development in Clostridium. Anaerobe 41, 104-112. doi: 10.1016/j.anaerobe.2016.05.011

Nakamura, Y., Gojobori, T., and Ikemura, T. (2000). Codon usage tabulated from international DNA sequence databases: status for the year 2000. Nucleic Acids Res. 28:292. doi: 10.1093/nar/28.1.292

Ng, Y. K., Ehsaan, M., Philip, S., Collery, M. M., Janoir, C., Collignon, A., et al. (2013). Expanding the repertoire of gene tools for precise manipulation of the Clostridium difficile genome: allelic exchange using pyrE alleles. PLoS One 8:e56051. doi: 10.1371/journal.pone.0056051

Peck, M. W., Fairbairn, D. A., and Lund, B. M. (1992). The effect of recovery medium on the estimated heat-inactivation of spores of non-proteolytic Clostridium botulinum. Lett. Appl. Microbiol. 15, 146-151. doi: 10.1111/j.1472765X.1992.tb00749.x

Perkins, W. E. (1965). Production of clostridial spores. J. Appl. Bacteriol. 28, 1-16. doi: 10.1111/j.1365-2672.1965.tb02121.x

Perkins, W. E., and Tsuji, K. (1962). Sporulation of Clostridium botulinum. II. Effect of arginine and its degradation products on sporulation in a synthetic medium. J. Bacteriol. 84, 86-94. doi: 10.1128/JB.84.1.86-94.1962

Purdy, D., O'Keeffe, T. A. T., Elmore, M., Herbert, M., McLeod, A., Bokori-Brown, M., et al. (2002). Conjugative transfer of clostridial shuttle vectors from Escherichia coli to Clostridium difficile through circumvention of the restriction barrier. Mol. Microbiol. 46, 439-452. doi: 10.1046/j.13652958.2002.03134.x

Roberts, T. A. (1965). Sporulation of Clostridium botulinum type E in different culture media. J. Appl. Bacteriol. 28, 142-146. doi: 10.1111/j.1365-2672.1965. tb02136.x

Roberts, T. A. (1967). Sporulation of mesophilic clostridia. J. Appl. Bacteriol. 30, 430-443. doi: 10.1111/j.1365-2672.1967.tb00321.x

Seys, F. M., Rowe, P., Bolt, E. L., Humphreys, C. M., and Minton, N. P. (2020). A gold standard, CRISPR/Cas9-based complementation strategy reliant on 24 nucleotide bookmark sequences. Gene 11:458. doi: 10.3390/ GENES11040458

Sobel, J. (2005). Botulism. Clin. Infect. Dis. 41, 1167-1173. doi: 10.1086/444507
Solomon, H. M., and Kautter, D. A. (1979). Sporulation and toxin production by Clostridium botulinum type G. J. Food Prot. 42, 965-967. doi: 10.4315/0362-028X-42.12.965

Solomon, H. M., and Lilly, T. J. (2001). "Clostridium botulinum” in Bacteriological analytical manual. U.S. Food and Drug Administration.

Strasdine, G. A., and Melville, J. (1968). Growth and spore production of Clostridium botulinum type $\mathrm{E}$ in chemically defined media. J. Fish. Res. Board Can. 25, 547-553. doi: 10.1139/f68-046

Tsuji, K., and Perkins, W. E. (1962). Sporulation of Clostridium botulinum 1. J. Bacteriol. 84, 81-85.

Tyrrell, E. A., MacDonald, R. E., and Gerhardt, P. (1958). Biphasic system for growing bacteria in concentrated culture. J. Bacteriol. 75, 1-4. doi: 10.1128/ JB.75.1.1-4.1958

Vlamakis, H., Aguilar, C., Losick, R., and Kolter, R. (2008). Control of cell fate by the formation of an architecturally complex bacterial community. Genes Dev. 22, 945-953. doi: 10.1101/gad.1645008

Whitmer, M. E., and Johnson, E. A. (1988). Development of improved defined media for Clostridium botulinum serotypes a, B, and E. Appl. Environ. Microbiol. 54, 753-759. doi: 10.1128/AEM.54.3.753-759.1988

Woods, D. R., and Jones, D. T. (1987). Physiological responses of Bacteroides and Clostridium strains to environmental stress factors. Adv. Microb. Physiol. 28, 1-64. doi: 10.1016/S0065-2911(08)60236-2

Zhang, Z., Dahlsten, E., Korkeala, H., and Lindström, M. (2014). Positive regulation of botulinum neurotoxin gene expression by CodY in Clostridium botulinum ATCC 3502. Appl. Environ. Microbiol. 80, 7651-7658. doi: 10.1128/AEM.02838-14

Conflict of Interest: The authors declare that the research was conducted in the absence of any commercial or financial relationships that could be construed as a potential conflict of interest.

Copyright (C) 2021 Mertaoja, Nowakowska, Mascher, Heljanko, Groothuis, Minton and Lindström. This is an open-access article distributed under the terms of the Creative Commons Attribution License (CC BY). The use, distribution or reproduction in other forums is permitted, provided the original author(s) and the copyright owner(s) are credited and that the original publication in this journal is cited, in accordance with accepted academic practice. No use, distribution or reproduction is permitted which does not comply with these terms. 